\title{
Unification of gravitation and electromagnetic force(second portion)
}

This paper was downloaded from TechRxiv (https://www.techrxiv.org).

LICENSE

CC BY 4.0

SUBMISSION DATE / POSTED DATE

$15-11-2021 / 18-11-2021$

CITATION

Zheng shengming, Physicist (2021): Unification of gravitation and electromagnetic force(second portion). TechRxiv. Preprint. https://doi.org/10.36227/techrxiv.17011199.v1

$\mathrm{DOI}$

10.36227/techrxiv.17011199.v1 


\title{
Unification of gravitation and electromagnetic force (Second Portion)
}

\author{
Zheng shengming（郑胜明）（Physicsworks@tom.com） \\ The contents of this article fall within Fields, Wave and Electromagnetics; Photonics and Electrooptics area of scope of Tech Rxiv, part \\ of it are published in my book (ISBN: 9789881986863 ) but is not published in journal. For convenience readers, I revise and submit it to here
}

Abstract: based on the new discovery of moving photon do create force and a formula of describe this new discovery: Applying this discovery from the micro world to the macro world, show that from atomic world to the galaxy world, the nature has been working obeys this law, and their actions can all be described by this formula. The Coulomb law and Newton law of university gravitation are only calculation formula in the specific condition status.

Keywords: Create Unification Physics discovery force, gravitation, electromagnetic force, unification.

Chapter.1. The validate of moving photon generate force

From before article: Gravitation origin ${ }^{[1]}$, Unification of gravitation and electromagnetic force ${ }^{[2]}$.we know that moving photons generate force. According to this validated discovery: we can know that there is interaction force between two light beams. Namely the distributing of light beam in space give rise to a force field, thus if change the force field, it will change the distributing of light beam in space; on the other hand, change the distributing of light beam will change the force field in space. Test this infer can further test validate of new discovery, for this, I do below experiment. See picture a of Figure1, a light source is created. When all light beam moving forward, the form of center light beam is difference with the form when only the center light beam moving forward. If only the center light beam moving forward, the center light beam increase more intensity than that when it with others light beams, this showing when other light beams do not moving forward; the more light beams have been attracted to the center. See picture $\mathbf{a , b}, \mathbf{c}, \mathbf{d}, \mathbf{e}, \mathbf{1 , 2 , 3 , 4 , 5}$. This experiment has come true validate of above discovery.

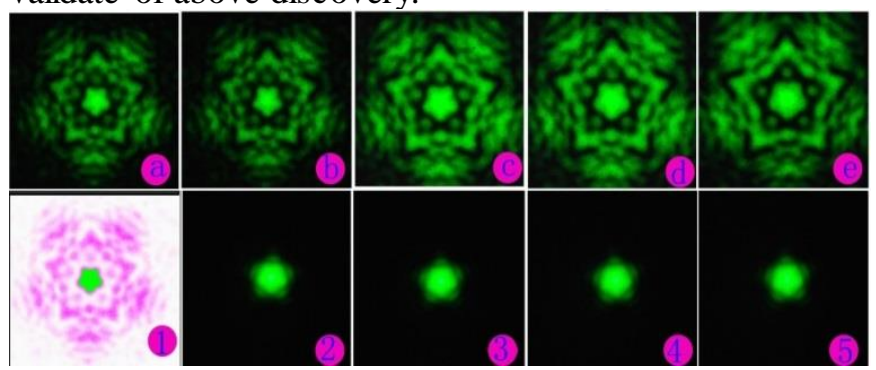

Figure1. Picture a is a light source. When all light beams moving forward, the form of center light beam is difference with the form when only the centers light beam moving forward. If so, the center light beam increases intensely, this indicates when other light beams do not move forward; the more light rays have been attracted to the center. see picture $\mathbf{a}, \mathbf{b}, \mathbf{c}, \mathbf{d}, \mathbf{e}, \mathbf{1 , 2}, \mathbf{3}, \mathbf{4}, \mathbf{5}$. This experiment tests the true of validate of above discovery that moving photons generate force. From above experiments, we can get a law of nature that the moving photon do creates force.

\section{Chapter.2. The quantitative experiment}

In before article: Gravitation origin ${ }^{[2]}$, we get below formula: $\vec{F}_{a}=\frac{m_{1} m_{2} \times \vec{v}_{1} \times\left(\vec{v}_{2} \times \vec{r}_{12}\right)}{4 \pi \Theta r_{12}^{3}} G_{a}$

About describing of this effect, see Gravitation origin ${ }^{[2]}$, here do not repeat it.

\section{Chapter.3. Applying in micro world}

\subsection{The electric charge of particle}

We now know that moving photons do generate force, and know the unification of electromagnetic force and gravitation, this suggest that if the particle holds photons are more or less than threshold of its internal balance of force, it will attract or repel other particle, hence it appears phenomenon of attract or repulse other particles, this showing the electric characteristics, this is the essence of electric charge of particle. From this we know the origin of electric charge of particle.

\subsection{Reveal Bohr hydrogen spectrum formula}

In hydrogen atom, one electron revolve around nuclear moving, between them, when the electron moving from velocity $v_{1}$ to $v_{2}$, and the distance between them from $r_{1}$ to $r_{2}$, in this changing process, the change of energy of electron is below:

$\Delta E=\int_{r_{1}}^{r_{2}} d r \int_{v_{1}}^{v_{2}} \frac{M V m v}{4 \pi \theta r^{2}} G_{a} d v=\int_{r_{1}}^{r_{2}} \frac{Q e}{r^{2}} k d r$

$\frac{M V m G_{a}}{4 \pi \theta} \times \frac{r_{1}-r_{2}}{r_{1} r_{2}} \times\left(v_{2}^{2}-v_{1}^{2}\right)=\frac{Q e k}{r_{1} r_{2}}\left(r_{1}-r_{2}\right) \backslash$

$v_{2}^{2}-v_{1}^{2}=\frac{4 \pi \Theta Q e k}{M V m G_{a}}$ Can get below formula:

$m \times\left(v_{2}^{2}-v_{1}^{2}\right)=m \times \frac{4 \pi \theta Q e k}{M V m G_{a}} ;$

$E_{2}-E_{1}=m \times \frac{4 \pi \Theta Q e k}{M V m G_{a}} \quad$ (1)

Meanwhile according to $\frac{M V m v}{4 \pi \theta r^{2}} G_{a}=\frac{Q e}{r^{2}} k$ consider $v=\frac{k_{\lambda}}{\lambda}$ Can get below formula: $v=\frac{4 \pi \Theta Q e k}{M V m G_{a}}=\frac{k_{\lambda}}{\lambda}$ Compare (1), eventually get below formula: 
$\frac{k_{\lambda}}{\lambda}=\frac{1}{m} \times\left(E_{2}-E_{1}\right) \quad, \quad$ Namely: $\quad \frac{1}{\lambda}=\frac{1}{m k_{\lambda}} \times\left(E_{2}-E_{1}\right)$ $m$ and $k_{\lambda}$ are all constant, can get: $\frac{1}{\lambda}=k \times\left(E_{2}-E_{1}\right)$.

This formula shows the reason why Bohr formula can describe the hydrogen spectrum. From this, in generalization: we can know the two body system can all be described by this formula. like the spectrum of hydrogen, and the spectrum of Hydrogen-like systems: include the ions $\mathrm{He}^{+}, \mathrm{Li}^{2+}, \mathrm{Be}^{3+}, \ldots \mathrm{U}^{91+}$, and the spectrum of Alkali metal. The Hydrogen is only specific case.

\subsection{The decay of particle}

According to above discovery that moving photons generate force, so there is force between photon and other particles. Namely, photons can interact with any other particles. One photon can be attracted or repelled by other photons or other particles. On the other hand, the size of force which moving photons created is specific. Two photons produce electron position pair need sufficiently energy, the appearing character of particle is determined by its moving state, if one particle absorbed one photon, its internal original balance of force will be changed, this will lead to fusion or fission for new balance of force, in this process, the parent particle displays decay process. Like below some examples: neutron decay, Mesons decay, nuclear decay etc. there are many photons in space, and any particle can absorb photon in any time, thus almost particle show decay character. Here know the reason why particles decay, it is due to the force between photons and particles.

\subsection{The mechanism of chemical reaction}

Applying in chemical reaction, see article Gravitation origin ${ }^{[1]}$, here need not repeat it.

\subsection{Applying in thermoelectricity}

Applying in thermoelectricity, see article Gravitation origin ${ }^{[1]}$, here need not repeat it.

From above five applying example, we can know that the formula $\vec{F}_{a}=\frac{m_{1} m_{2} \times \vec{v}_{1} \times\left(\vec{v}_{2} \times \vec{r}_{12}\right)}{4 \pi \Theta r_{12}^{3}} G_{a}$ is the universal law in the micro world.

\section{Chapter.4. Applying in macro world}

\subsection{The essence of bending of light}

From above we have known the origin of gravitation. Here use this finding to calculate the bending of light. Below Figure 2 is the state of light of star passing near the Sun, $r$ is the distance between center of Sun and passing light. $\mathrm{A}$ is the actual site of star, B is the site of seeing in the earth, $\mathrm{R}$ is the radius of Sun. In Figure2: according to formula: $\vec{F}_{a}=\frac{m_{1} m_{2} \times \vec{v}_{1} \times\left(\vec{v}_{2} \times \vec{r}_{12}\right)}{4 \pi \Theta r_{12}^{3}} G_{a}$ Can get: $F_{a}=\frac{m_{s} m_{p} v_{s} c}{4 \pi \theta r^{2}} G_{a}$, where $m_{s}$ is the mass of the Sun, $v_{s}$ is the average speed of moving particle in the Sun, $m_{p}$ is the mass of photon, $c$ is the speed of light. $r$ is the distance between center of Sun and passing light. Because the gravitation that star light accepted from Sun is changed along with the distance changing, and the accepted gravitation is the biggest size of value when star light is passing the scope where nearest Sun. So in this special scope of nearest the Sun that light moved distance $S$ play key role for bending of light, in this traveled distance, light accepted the gravitation may represent the gross gravitations that accepted in its whole traveled path. Then after light has passed side of Sun it can got an increased velocity by this gravitation:

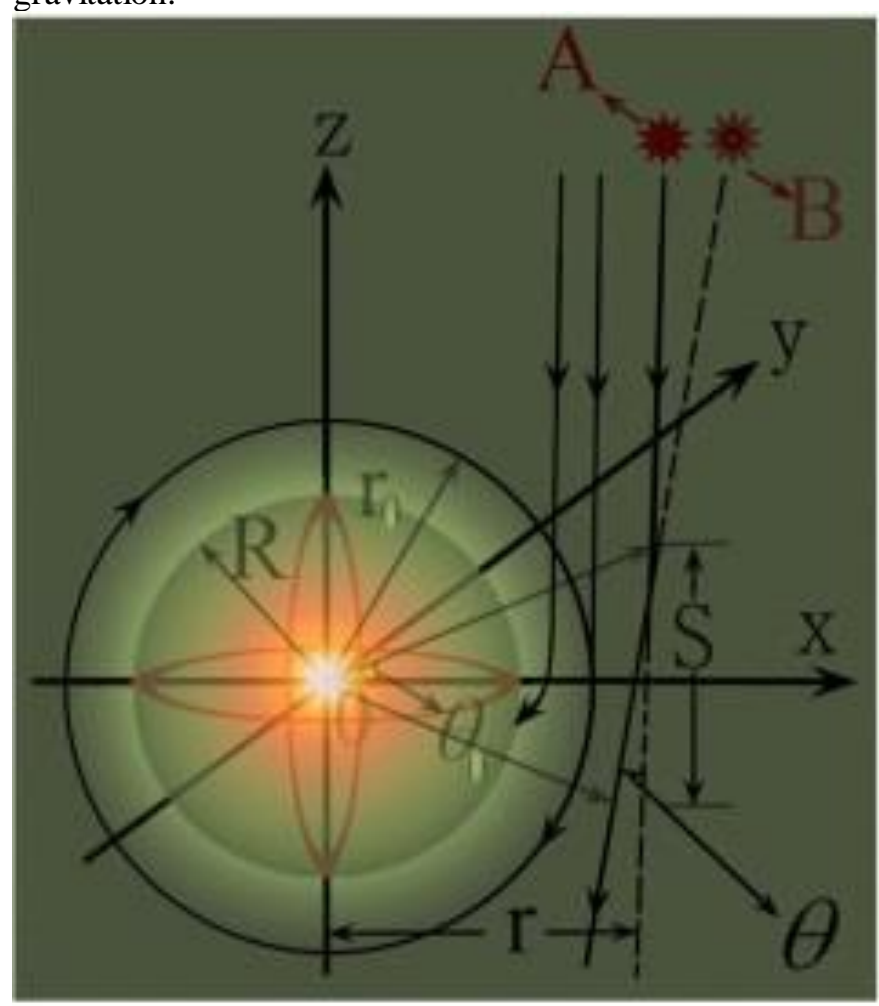

Figure 2. This is the state of light of star passing near the Sun, $r$ is the distance between center of Sun and passing light. $\mathrm{A}$ is the actual site of star, $\mathrm{B}$ is the site of seeing in the earth, $\mathrm{R}$ is the radius of Sun.

$v=a t=\frac{m_{v}, m_{p} c}{r^{r} m_{p}} G \cdot \frac{s}{c}=\frac{m_{v} v_{s}}{r^{2}} G_{a} S=\frac{m_{s} v_{s}}{r^{2}} G_{a} 2 r \operatorname{tg} \theta_{0}=\frac{m_{s} v_{s}}{r} G_{a} 2 \operatorname{tg} \theta_{0}$

Namely, $v=\frac{m_{s} v_{s}}{r} G_{a} 2 t g \theta_{0}$. When the light arrive the earth, it got a displacement $h: \quad h=v t=\frac{m_{s} v_{s}}{r} G_{a} 2 \operatorname{tg} \theta_{0} \frac{L_{1}}{c}=\frac{m_{s} v 2 \operatorname{tg} \theta_{0} G_{a}}{r c} L_{1}$;

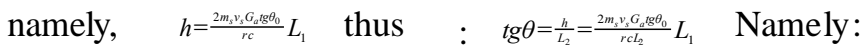
$\operatorname{tg} \theta=\frac{2 m_{y}, G_{d t g} \theta_{0}}{r c L_{2}} L_{1}$. Where $L_{1}$ is the distance between earth and Sun, $L_{2}$ is the distance between earth and star, because the $m_{s}, v_{s}, G_{a}, c, L_{1}$ are all constant, only $L_{2}, \theta_{0}$ have a little change, $\operatorname{sotg} \theta=\frac{h}{L_{2}}=\frac{2 \lg \theta_{0}}{r L_{2}} k$, here if $L_{2}$ and $\theta_{0}$ are all constant, then $\operatorname{tg} \theta=\frac{2 \operatorname{sg} \theta_{0}}{r t_{2}} k=\frac{k_{b}}{r}$. Namely: $r \operatorname{tg} \theta=k=$ constant. About result of test, see below table1

Table 1:

\begin{tabular}{cccc}
\hline$r$ & $\theta$ & $\operatorname{tg} \theta$ & $\operatorname{rtg} \theta$ \\
\hline 1.85 & $0.95^{\prime \prime}$ & $4.61 \mathrm{E}-06$ & $8.52 \mathrm{E}-06$ \\
4.82 & $0.37^{\prime \prime}$ & $1.79 \mathrm{E}-06$ & $8.65 \mathrm{E}-06$ \\
7.05 & $0.26^{\prime \prime}$ & $1.26 \mathrm{E}-06$ & $8.88 \mathrm{E}-06$ \\
8.35 & $0.21^{\prime \prime}$ & $1.01 \mathrm{E}-06$ & $8.50 \mathrm{E}-06$ \\
8.5 & $0.215^{\prime \prime}$ & $1.04 \mathrm{E}-06$ & $8.85 \mathrm{E}-06$ \\
\hline
\end{tabular}


Above Table1 is the examined result of deduced conclusion. From this test we can find that formula $\operatorname{tg} \theta=\frac{2 m_{s} v_{s} G_{a} \operatorname{tg} \theta_{0}}{r c c_{2}} L_{1}$ is compatible with the data of bending of light when it passing near the Sun.

According to formula: $\vec{F}_{a}=\frac{m_{1} m_{2} \times \vec{v}_{1} \times\left(\vec{v}_{2} \times \vec{r}_{12}\right)}{4 \pi \theta r_{12}^{3}} G_{a}=m_{p} \frac{c^{2}}{r} \quad$ Can get three status of bending of light when it is passing side of Sun: First status when $\frac{m_{s} v_{s}}{4 \pi r}>c$ : the light beam will be absorbed by Sun. In earth cannot see the light beam. Second status when $\frac{m_{s} v_{s}}{4 \pi r}=c$ : the light beam will revolve around the Sun. In earth cannot see the light beam. Above two conditions indicate that in special scope of nearest the Sun, we cannot see the star which in the sky. Third status when $\frac{m_{v} v_{s}}{4 \pi r}<c$ : the light beam will moving forward, and in earth can see this bending of light. These three statuses are in good agreement with the fact that in earth see phenomena. Meanwhile from these three statuses, we can clear know the star light passing what scope of nearest the Sun where the star light cannot reach earth, know the reason why we cannot see star when its light is passing nearest the Sun, and know the orbit of light moving in the space. All these information, the General relativity is unable tell us.

\subsection{The dark matter does not exist}

4.2.1.The gravitation of rotation of spiral galaxy

Observations display the spiral galaxy like the rotation of disk. It includes two features: one is the spiral arm. Second center spherical component includes a large halo and the nuclear bulge, where concentrated main mass of galaxy.

According to formula: $\vec{F}_{a}=\frac{m_{1} m_{2} \times \vec{v}_{1} \times\left(\vec{v}_{2} \times \vec{r}_{12}\right)}{4 \pi \Theta r_{12}^{3}} G_{a}$. For a spiral galaxy there is a central bulge where most of the mass is concentrated and the spiral arms are spread over a disk. For a star in such a galaxy at a distance $r$ from the galactic center moving with a circular velocity: $v$. When $M_{b o d y<r}$ : where $M_{<r}$ is the mass enclosed within the radius $r$. If the star is within the dense central region (or central hub) of the galaxy, then $M_{<r}=\frac{4}{3} \pi r^{3} \rho$, where $\rho$ is the average density of the central hub. Therefore, with in the central hub one expects from

$$
\frac{m M v V}{4 \pi r^{2}} G_{a}=\frac{m_{3}^{4} \pi r^{3} \rho \cdot 2 \pi r V}{4 \pi r^{2} T} G_{a}=\frac{m v^{2}}{r} \text {. (consider: } v=\frac{2 \pi r}{T} \text { ) }
$$

Can get: $v=\sqrt{\frac{2 \rho \pi G_{a} V}{3 T} r^{3}}=\sqrt{r^{3}} \sqrt{\frac{2 \rho \pi G_{a} V}{3 T}}$

When $M_{b o d y>n} M>r: \frac{m M v V}{4 \pi r^{2}} G_{a}=\frac{m v^{2}}{r}$ consider: $v=\frac{2 \pi r}{T}$

Can get: $\frac{m M V 2 \pi r}{4 \pi r^{2} T} G_{a}=\frac{m v^{2}}{r}$ namely: $v=\sqrt{\frac{M V G_{a}}{2 T}}$. In this result, rotation velocity of spiral galaxy is a constant when the $\sqrt{\frac{M V G_{a}}{2 T}}$ is constant, this is in extremely good agreement with that the observational measurements of rotation curves for several spiral galaxies show $v$ equally constant for large $r$. Certainly these results expose that the Newton's law of universal gravitation is not natural law, the dark matter does not exist in nature.

According to formula: $\vec{F}_{a}=\frac{m_{1} m_{2} \times \vec{v}_{1} \times\left(\vec{v}_{2} \times \vec{r}_{12}\right)}{4 \pi \Theta r_{12}^{3}} G_{a}$ get two results:

when $\mathrm{M}_{<\mathrm{r}}: v=\sqrt{r^{3}} \sqrt{\frac{2 \rho \pi G_{a} V}{3 T}} ;$ when $\mathrm{M}_{>\mathrm{r}}: \quad v=\sqrt{\frac{M V G_{a}}{2 T}}$. According to formula: $F=\frac{m_{1} m_{2}}{r^{2}} G$, can get below two results: when $\mathrm{M}_{<\mathrm{r}}: \quad v=\sqrt{\frac{4 \pi \rho G}{3}} r$; when $\mathrm{M}_{>\mathrm{r}}: v=\frac{\sqrt{M G}}{\sqrt{r}}$. It is unequivocal that the two results which from formula $\vec{F}_{a}=\frac{m_{1} m_{2} \times \vec{v}_{1} \times\left(\vec{v}_{2} \times \vec{r}_{12}\right)}{4 \pi \Theta r_{12}^{3}} G_{a}$ are in extremely good agreement with observational facts, but the two results from $F=\frac{m_{1} m_{2}}{r^{2}} G$ are not in agreement with observational facts.

\subsubsection{The observation facts confirm discoveries}

According to $v=\sqrt{\frac{M V G_{a}}{2 T}}$. in one galaxy: if the $\frac{M V G_{a}}{2 T}$ is constant, and the main mass of galaxy concentrated in the center of galaxy, the rotation curve of spiral galaxy will be a flat, if period will be changing larger than mass's changing, the rotation curve of spiral galaxy will be changed too. Based on these, because the N4565, N4594, M31, N891 around center of galaxy rotation like the standard of disk rotation, from their center to large $r$, the $\frac{M V G_{a}}{2 T}$ is close constant, we can infer that the velocity of rotation will be constant along with increasing radii. The curve rotation is flat. Among these spiral galaxies, the $\mathrm{N} 4565$ is the most standard of rotation disk, thus its rotation curve appear a straight line. The N5033 has main mass in its center, begin with standard rotation disk, then change this status, so its rotation curve is flat first, then decrease. The M83, N7217, M51, M81, and our galaxy is not standard of rotation disk, their rotation curve is not flat. The observation fact show in good agreement with these conclusions ${ }^{[3]-[10]}$.

4.2.3. Newton's law of universal gravitation is not natural law

By above presenting facts compare with the calculated result which from Newton's law of universal gravitation, show Newton's law is not in agreement with observational some facts, so appear hypothesis of dark matter. We now know that there is not dark matter in nature, in fact the Newton's law of universal gravitation laws is based on the Kepler law. Namely the Newton's law of universal gravitation is not the law of nature, it is only an approximation formula which deduced by Kepler's third law to calculate some mathematics problem. see below several observational facts [4], [10] these observational rotation curves show Newton's law of universal gravitation is not in agreement with the observational facts. But above conclusion of discoveries is in extremely good agreement with the observational facts. The dark matter does not exist.

Above observations facts also display that the highest site( the $\mathrm{O}$ point in rotation curve) of rotation curve from 
observation is higher than the highest site( the $\mathrm{N}$ point in rotation curve) from Newtonian law calculated result, this is in good agreement with that the calculated speed value by $\quad v=\sqrt{r^{3}} \sqrt{\frac{2 \rho \pi G_{a} V}{3 T}} \quad$ which from $\vec{F}_{a}=\frac{m_{1} m_{2} \times \vec{v}_{1} \times\left(\vec{v}_{2} \times \vec{r}_{12}\right)}{4 \pi \Theta r_{12}^{3}} G_{a}$ is larger than calculated speed value by $v=\sqrt{\frac{4 \pi \rho G}{3}} r$ which from $F=\frac{m_{1} m_{2}}{r^{2}} G$ Thus Newton's law of universal gravitation is wrong that for describe the galaxy.

On the other hand,, other observations, like gravitational lensing - the bending of light from distant sources by the cluster's gravity-also confirm that moving photons generate gravitation, but is not due to dark matter.

\subsection{Demonstrate Kepler third laws}

When Sun and Planet are all moving forward, they are all revolving around the center of mass moving, in this condition: the Sun and all Planets are all around their center of mass moving forward in the infinity universe, this is as same as they are all moving in one space, so here need not consider the characteristic of medium: $\boldsymbol{\Theta}$, thus:

$F_{a}=\frac{M V^{2} m_{p} v_{p}}{4 \pi r_{c}^{2}} G_{a}=M_{p} \frac{v^{2}}{r_{c}}$, Consider: $M_{p}=m_{p} v_{p} k$

Can get: $v^{2} r_{c}=\frac{M V^{2} G_{a} k}{4 \pi}$ in the Sun system, because the $M V k G_{a}$ are all constant, so $v^{2} a=$ constant, this is Kepler third laws. Please note: here the value of $M$ is not equal the mass of we have known Sun mass.

\subsection{Demonstrate Newtonian law of universal gravitation}

Demonstrate Newtonian law of universal gravitation, see article Gravitation origin ${ }^{[1]}$, here need not repeat it.

\subsection{The Universe does not inflation!}

\subsubsection{The Doppler Effect in light.}

In light: according to above discovery, we now know the light is not wave-particle duality, it is a particle, according to formula: $\lambda v=k_{\lambda}$ we can get below formula: $\lambda=\frac{k_{\lambda}}{v}$, namely the wavelength is the reciprocal of speed, for observer, some photons moving tow ard to left observer A, some photons moving toward to right observer B, so in observer A eye, the wavelength of photon is $\frac{k_{\lambda}}{v-v_{0}}$, where $v$ is the speed of light, $v_{0}$ is the speed of atom, in this case the wavelength is increased, this is the red shift. The wavelength of photon tow ard to observer B is $\frac{k_{\lambda}}{v+v_{0}}$, in this case the wavelength is decreased, and this is blue shift. Namely, the frequency of photons is decreased when it moving toward to left observer $A$, the frequency of photons is increased when it toward to observer B. This is reason of red shift and the blue shift. But the status of atomic moving which sent light did not change.

\subsubsection{The Universe does not inflation}

Doppler Effect in Astronomy. According to above analyze, because some stars depart from earth, its wavelength which earth received is $\frac{k_{\lambda}}{v-v_{0}}$, where $v$ is the speed of light, $v_{0}$ is the speed of star, and some stars coming to earth, its wavelength which earth received is $\frac{k_{\lambda}}{v+v_{0}}$, so we can observe the red shift of light of some stars and the blue shift of some stars! Why we observe the light from farther star, its more red shift than near star sent, I think this like the moving of bullet, because in moving process, the more moved distance, the more lose energy, so the lower speed of it! This is the reason why the longer distance between earth and star, the more red shift appears! Because the more distance between earth and star, the more distance need move for light, so its speed will be more decreased than nearer the earth stars sent. in fact in this state, only received the wavelength of light will be changed in difference distance between earth and star, but the state of moving star do not change as same as above observers A and B (see the state of moving car), and the space also does not change. Thus the Universe is not inflation.
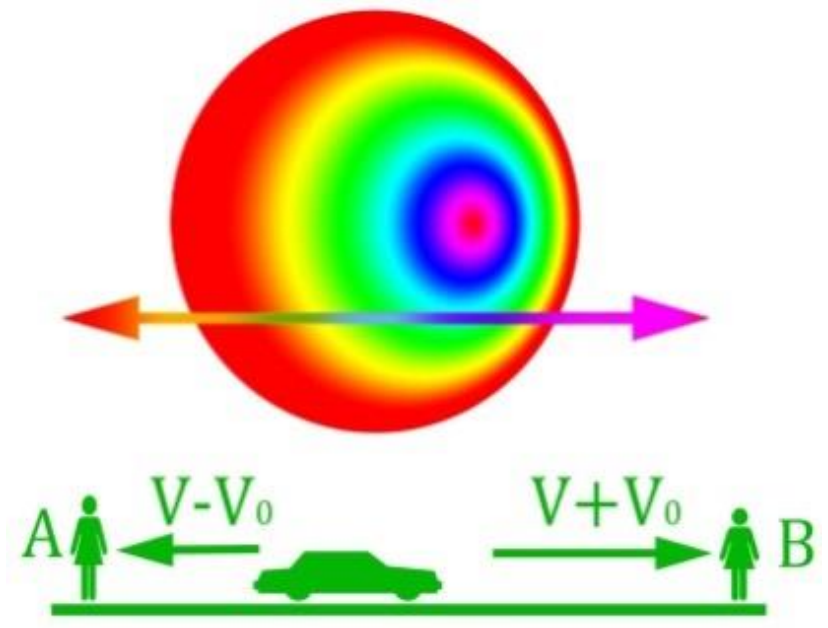

Figure.3. this figure show the es sence of Doppler effect In figure 3 , if the speed of car is $v_{0}$, the speed of sound of car is $v$, when the car is moving tow ard right observer $\mathrm{B}$, for observer A, she receive the speed of message is $v-v_{0}$, where $v_{0}$ is the speed of car, $v$ is the speed of sound. For observer $B$, she receive the speed of message is $v+v_{0}$, so the observer A receive the frequency of sound is lower than car sent, the observer $B$ receive the frequency of sound is higher than car sent, in fact the frequency of sound which car sent did not change, only the observer received frequency is changed! This is the essence of Doppler Effect.

\section{Chapter.5. Conclusion}

We have known the essence of electric charge and the essence of gravitational mass in atom; meanwhile we have also known the unification of gravitation and electromagnetic force by the way of Applying this new 
discovery from micro world to macro world, about the more contents and the more evidences of unification of the electromagnetic force and gravitational force, please waiting to see will be coming next article ${ }^{[11],[12]}$.

\section{Ack nowledgment}

I express my appreciation sincerely to Yang Zhenfeng who is in the institute of Mathematic, and my friend Shao shengtang, Jiu guoqiang, Wang xuezheng, Xi xinghua, and Dong qianlin, my middle school teacher Zhang linxiang. My young uncle sister Gou susheng. My young brother Zheng guoman, My young sister Zhnegjunfang. They all gave me a lot of spiritual and financial support when I did the research about this paper. I also express my gratitude for my father Zheng heizi, who gave me great love, and that is the driving force of my moving forward.

\section{References}

[1] shengming, Zheng (2021): Grativation origin. TechRxiv. Preprint. https://doi.org/10.36227/techrxiv.16677298.v1

[2] shengming, Zheng (2021): Unification of gravitation and electromagnetic force(First Portion). TechRxiv. Preprint. https://doi.org/10.36227/techrxiv.16817275.v1

[3] Mike Seeds, Dana Backman. Astronomy: The Solar Sy stem and Beyond, p.349

[4] Malcolm S. Longair. Galaxy Formation p.68

[5] Marc L. Kutner Astronomy:A Phy sical Perspective

[6] H. Karttunen, P. Kröer, H. Oja, M. Poutanen, K. J. Donner Fundamental Astronomy.

[7] MARIO LIVIO The Dark Universe: Matter, Energy and Gravity p,

[8] MARIO LIVIO The Dark Universe: Matter, Energy and Gravity p, 4.

[9] Riccardo Scarpa Modified Newtonian Dynamics, an Introductory Review p.3.

[10] MARIO LIVIO The Dark Universe: Matter, Energy and Gravity p, 69 .

[11] Zheng sheng ming. Mechanism of interaction in moving matter-the origin of gravitation. ISBN 9783847326588.2011.

[12] Zheng sheng ming. Nature mechanism-force origin.ISBN 97898819868632012 